\title{
Enhanced channel zapping procedure for better QoE in IPTV services
}

\author{
Jyoti \\ M-Tech Student, Department of CSE, Advance Institute of Technology and Mgt, Palwal, Haryana, India \\ Mahesh Singh \\ India 1 Assit. Prof., Department of CSE, Advance Institute of Technology and Mgt. Palwal, Haryana, India
}

\begin{abstract}
Internet Protocol TV (IPTV) deployments in the consumer electronics market is developing among the people in last some years. In IPTV, Quality of Experience (QoE) is very serious factor for end-subscriber satisfaction. Future and available IPTV systems will provide a large no. of channels to subscribers. The most significant factors that have effects on QoE in IPTV systems are channel selection and channel zapping problems. Normally, the channel zapping time is based on various parameters i.e. network delay time, Internet Group Membership Protocol (IGMP) command processing time, IPTV consumer device processing delay time, MPEG decoder time, jitter buffer delay time and Conditional Access/Digital Rights Management (CA/DRM) stoppage period. In this paper, we show channel change delay analysis in IPTV systems and we introduce a novel mechanism depending on channel-based peer selection, known as CBPS where the most proper peer to build peer-to-peer (P2P) exchanging information will be found from a look-up table in the central server.
\end{abstract}

Keywords -Channel zapping times; Peer-to-Peer (P2P) communication, Quality of Experience (QoE), Internet Protocol TV (IPTV);

\section{INTRODUCTION}

IPTV deployments become very famous with its benefits in the users electronics market in last some years. IPTV is a novel form of digital television technique. In this system, digital television facilities are provided to consumers throughout a network infrastructure by utilizing Internet protocol [1]. IPTV system utilizes the digital video compression method to deliver the multimedia content to end-subscribers. Quality of Experience (QoE) is the most significant factor to fulfill the IPTV consumers. Customers want to use the IPTV channels very frequently and see them to be non-blocking, smooth and without any disturbance. From this point of view, QoE is the most significant parameter for IPTV service suppliers to satisfy.

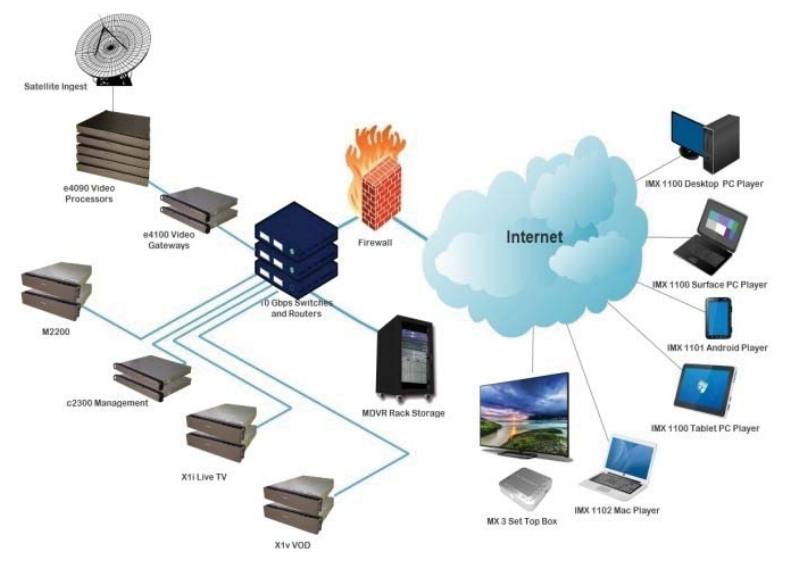

Channel zapping time is assumed as key factor for QoE metric of multicast-based IPTV systems [2]. Channel zapping time can be described as the time difference between the currently seen channel and the display of the first frame of the new required channel by end-subscriber on the TV screen. So thus, the reliable and frequent channel zapping time is the most significant element in validation of QoE in multicast-based IPTV systems. IPTV service suppliers will provide thousands of channels to their consumers. 
In the literature review, there are several papers that cover channel zapping times [3-6]. Among the introduced mechanisms in the previous works that we analyzed, various methods from utilizing multiple unicast streams to add adjacent channels [3], employing small Group of Pictures (GOP) size [4], decreasing IGMP command processing delay and predictive tuning by subscriber's channel selection behaviors [5] are showed. In [6], the authors introduced the technique that decreases channel zapping time by forwarding probe message to peers. With respect to this technique, the device that requires channel change will forward to a probe message to other network peers. Then the other peers that see the required channel again will forward a message to channel switched peer. According to this method [6], several messages have to be signaled among peers in the IPTV network at the time of channel change. When it is considering that there will be several peers in IPTV multicast groups, the message signaling technique will not be efficient. If mostly peers in the multicast group perform the channel zapping process at the simultaneously, the message overhead can happen in the system and furthermore a messaging delay during receiving/sending messages among peers will occur. In this paper, we suggest peer-to-peer transmission by CBPS peer selection algorithm among peers in the IPTV multicast-based system to decrease channel zapping times. With our suggested technique, when the zapping process is began by end-user, if the novel tuned channel is not existed in home gateway (HG), the peer partner between all peers watching the new required channel in the IPTV multicast group will be found by performing channel-based peer selection algorithm and P2P fast communication will be demonstrated between channel switched IPTV user device and it's peer partner to obtain the novel tuned channel. The information about the peer partner that will build a P2P communication with channel zapped device is hold in a look-up table in IPTV central server. When a device begins to zap process, it will provide this information. With the introduced algorithm, the time required for IGMP command buffering and process for the new channel will be eliminated. These operations will be continued in the background and end-subscriber will not wait to process for these. At zapping time, end-subscriber will view the channel from its peer partner. So the channel zapping time will be reduced for IPTV multicast systems to about 1-2 sec. by utilizing our suggested algorithm. In comparison of work in [6], also the messaging overhead in the system will not happen. The remaining paper is presented as follows. In Section II, the channel switching process in IPTV systems and the parameters affecting channel change time are presented. And the available methods for decreasing channel zapping time are explained in Section III. Section IV offers the suggested system to decrease channel zapping time is showed. At last, conclusions are presented in Section V.

\section{PROPOSED WORK}

\subsection{PROPOSED APPROACH}

In this section, we suggest peer-to-peer communication between the channel zapping IPTV device and the other peer that will be found by utilizing channel-based peer selection algorithm on IPTV network to solve the problem of IGMP command processing delay at the time of the channel zapping period in multicast-based IPTV systems. To choose the peer partner, a look-up table on the IPTV server will be hold and managed at the regular interval. Then this information will be forwarded to the channel zapped IPTV device at the zapping mode. When it obtains the information regarded to its peer partner that watches the required channel and is in the closest hop count away, the peer-to-peer communication will be begun between channels zapped device and its peer partner. At zapping time, it will receive unicast stream from its peer partner. By this technique, the required time to process IGMP command will be eliminated and so the zapping time will be reduced from $5 \mathrm{sec}$ to $1 \mathrm{sec}$. Fig. 2 shows the introduced system to decrease the zapping times. In this fig, L1, L2, L3, L4, L5 represents the distance as a hops count among peer-topeer nodes. Peer selection algorithms are categorized into two classes as global algorithms and user algorithms. The algorithms in the first group will be positioned in receiver side and will run on every peer in the IPTV multicast group or peer-to-peer systems. The second group algorithm will run in centralized server and the peer selection control will be done on server. In our method, we will utilize channel-based peer selection algorithm executing on IPTV central server. In this technique, the channel information on the watching mode and hops count information for every peer in the IPTV network will be gathered in the look-up table as called Dynamic Hash Table (DHT) on the IPTV server. The information about the peers (viewing/zapping/standby mode) mode, the currently viewed channel by the peers in the IPTV network and hops count among nodes will be involved in the table and it will be managed at the regular interval. In channel zapping process in IPTV systems, it is considered that TV viewer currently tune the channel1 (CH1) at XXX.XXX.XXX.6 IP multicast address and requests to switch the channel2 $(\mathrm{CH} 2)$ at the XXX.XXX.XXX.9 IP multicast address. In this situation, IPTV device firstly will forward an IGMP Leave message and then IGMP Join message for new required channel to HG. If the new required channel to tune is not existed in HG, the message involving the information about the peers that watches currently a novel zapping channel will be forwarded to channel zapped IPTV device. 


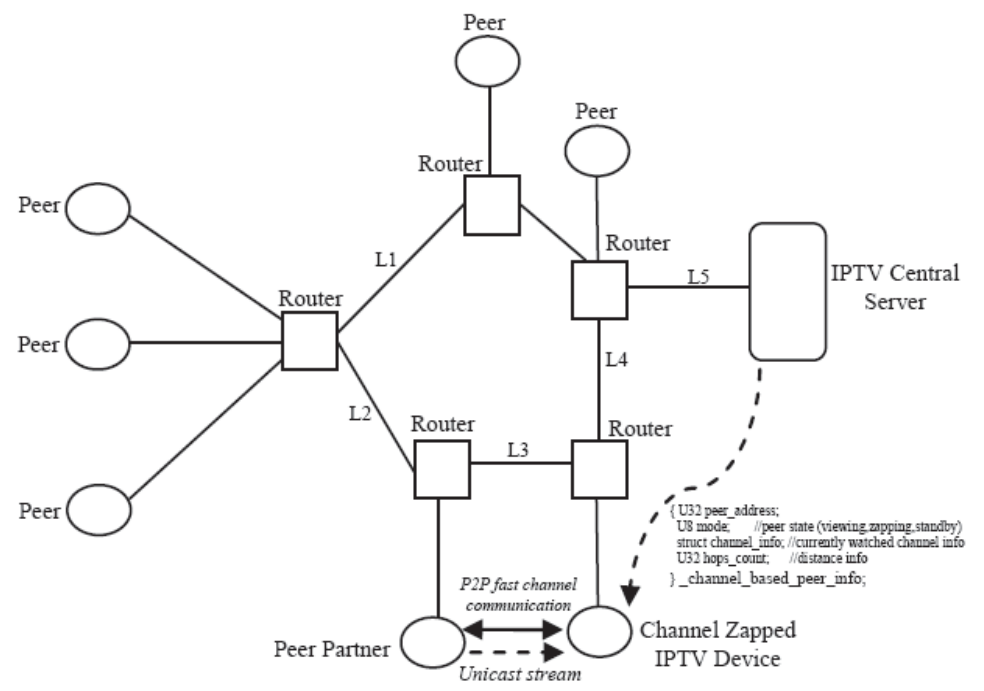

Fig. 2: The proposed system for reducing the channel zapping time

In the look-up table on the server, it will be hold the distance information as a hops count for each peer with the channel information. As soon as the channel switched IPTV device obtains this message involving the peer information that watches currently the required channel and also its distance (for example 2 or 3 hops away) between the channel switched IPTV device and the peer partner, it will attempt to link its peer partner in the novel IP multicast group to build peer-to-peer communication with it in zapping process. While zapping mode, the channel switched IPTV device will obtain unicast stream from its peer partner by utilizing the demonstrated IP unicast channel communication. Until the data for the zapped channel to tune from native IP multicast reaches and is buffered well to play, the unicast stream will be obtained from peer partner. When the channel coming IPTV multicast server exist, IPTV user device will began to display it. By utilizing unicast stream for novel required channel from the peer partner, the new channel stream coming from peer partner will be existed very frequently on IPTV device in zapping mode. In this manner, the IGMP command processing delay problem during channel zapping mechanism will eliminate and so thus channel zapping delay in IPTV multicast systems will be decreased.

\section{EXPERIMENT AND RESULT}

This section presents the results obtained from implementing the simulation model. The parameters: Average Queuing Delay, Throughput and Utilization will be examined to illustrate the performance of Triple Play services over VDSL2 broadband access technology. The simulation model scenario indicates the transfer of Triple Play service using VDSL2 technology across 300 meters and 500 meters. This scenario clarifies two states of transmission: Triple Play services transmission within the building that contains the DSLAM which is over 300 meters, and the transmission over 500 meters presented by services transferring to another adjacent building, as shown in Fig. 6 earlier. VDSL2 downstream bit rate $(100 \mathrm{Mbps})$ reduces the effect of local loop length change due to distance increase; on the other hand, the Average Queuing Delay becomes much smaller while the Throughput becomes more stable during simulation time. The Average Queuing Delay and the Throughput are shown in Fig. 3. 


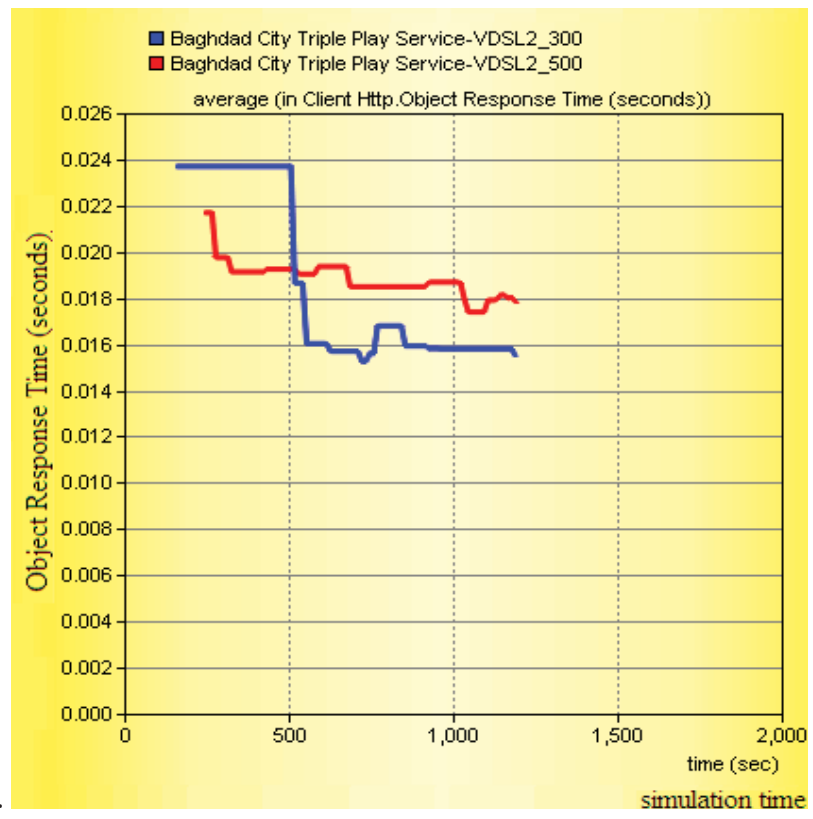

Fig 3: HTTP Object Response Time for the distance (300and 500Meter).

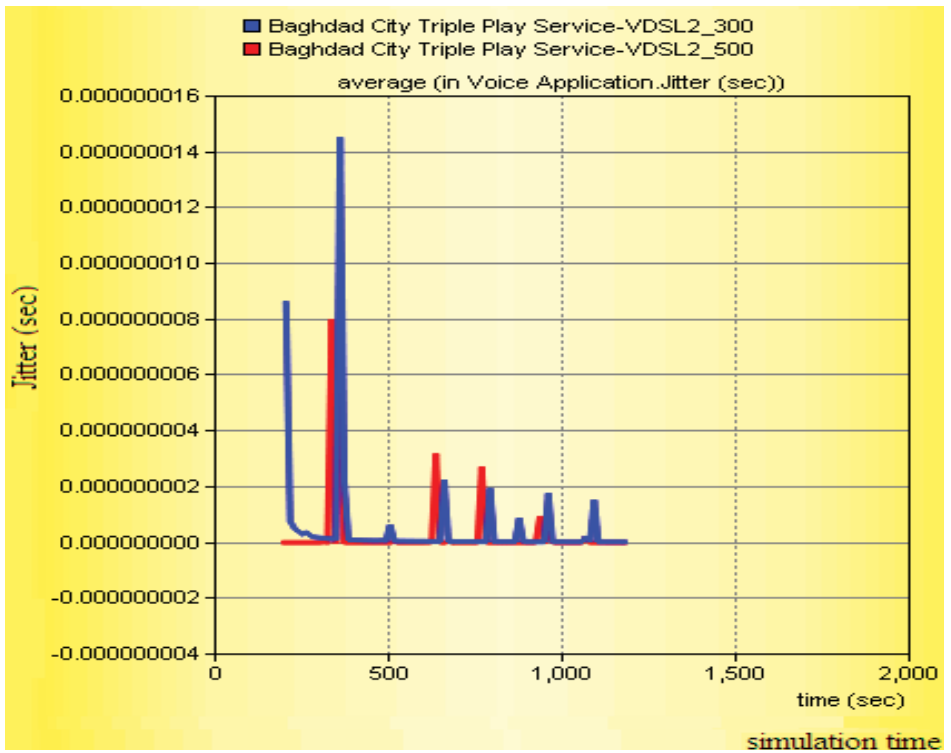

Fig 4: VOIP jitter for the distance (300 and 500 meter)

\section{IV.CONCLUSION}

In IPTV multicast systems, channel zapping time or channel switching delay is the most significant key element of Quality of Experience (QoE). To fulfill the IPTV end-subscriber, decreasing channel zapping delay is serious problem that is required to solve by IPTV service suppliers. Channel zapping delay for IPTV multicast facilities should be as short as channel zapping times in conventional broadcasting facilities i.e. terrestrial, cable and satellite. In this paper, firstly we enquired channel zapping times and channel zapping method in IPTV multicast systems. We showed the elements supporting channel zapping delay and we talked about the available techniques to decrease the channel zapping time. We suggested peer-to-peer communication by finding of peer partner utilizing channel-based 
peer selection Algorithm that is executing on the IPTV central server to eliminate IGMP command delay during channel zapping method and so decrease channel zapping delay.

\section{REFERENCES}

[1] A. Shehu, A. Maraj, and R. Mitrushi, "Analysis of QoS requirements for delivering IPTV over WiMAX technology", Proc. International Conference on Software, Telecommunications and Computer Networks (Soft- COM), pp. 380-385, 2010

[2] A. Bhunia, I. Misra, S. Sanyal, and A. Kundu, "Performance study of Mobile WiMAX network with changing scenarios under different modulation and coding”, International Journal of Communication Systems, 24, pp. 1087-1104, 2011.

[3] A. Aluwihare, J. Beckman, R. Flask, E. Kerch, J. Laferriere, M. Mekic, J. Nerschook, N. Parbhakar, T. Ward and J. Williams, "Triple Play Service Deployment: A Comprehensive Guide to Test, Measurement, and Service Assurance", JDS Uniphase Corporation, 2007.

[4] A. Rehman, T. Khan, and S. Chaudhry, "Study of WiMAX Physical Layer under Adaptive Modulation Technique using Simulink", International Journal of Scientific Research Engineering \& Technology, Vol.1, No.5, pp. 05-11, 2012.

[5] A. Abdennour, "VBR Video Traffic Modeling and Synthetic Data Generation Using GA-Optimized Volterra Filters", International Journal of Network Management, Vol.17, pp. 231-241, 2006.

[6] A. Rehman, T. Khan, and S. Chaudhry, "Study of WiMAX Physical Layer under Adaptive Modulation Technique using Simulink", International Journal of Scientific Research Engineering \& Technology, Vol.1, No.5, pp. 05-11, 2012.

[7] A. Shehu, A. Maraj, and R. Mitrushi, "Studying of different parameters that affect QoS in IPTV systems", Proc. International Conference on Telecommunications and Information (WSEAS), 2010

[8] A. Quadir, M. T. Arefin, and H. E. Sandström," Reliable IPTV Service Delivery Using PIM-SSM Routing ", Journal of Scientific Research (J. Sci. Res.), Volume 1, No.3, August, 2009. 